\title{
A modular system of software tools for multicriteria model analysis
}

\author{
J. Granat, T. Kreglewski, J. Paczyñski, A. Stachurski, A.P. Wierzbicki \\ Institute of Control and Computation Engineering \\ Warsaw University of Technology \\ Nowowiejska 15/19, 00665 Warsaw, Poland. \\ Tel/Fax: +48 22-253719. e-mail: J.Granat@ia.pw.edu.pl
}

\begin{abstract}
Applications of various analytical models in decision support systems motivate the development of efficient tools for the analysis of such models and their optimization. There are various approaches to the design of DSS based on analytical models. We focus here on developing well defined modular tools supporting selected phases of decision process, which can be used to build a DSS customized to a problem being solved.
\end{abstract}

\section{Keywords}

Modelling, decision support, multiobjective optimization

\section{INTRODUCTION}

Modular tools considered in this paper support selected phases of decision process. We assume that an analyst or an engineering designer uses substantive model expressing essential aspects of the decision situation.

The first set of tools supports the phase of model formulation and introductory model analysis. In this phase a user, usually an analyst, defines the substantive model and edits it on the computer. This phase was not explicitly supported in earlier versions of DIDAS-type systems (Lewandowski at al., 1983) and the user had to separately prepare (define and edit) his models, in nonlinear case together with user-supplied formulae for the derivatives of all outcome functions with respect to decision variables. It is a known fact that most mistakes in applying nonlinear programming methods are made when determining derivatives analytically; thus, this way of substantive model preparation required substantial experience in applications of nonlinear programming. The definition and edition of substantive models in the next generation of DIDAS-type systems, e.g. in IAC-DIDAS-N (Kręglewski at al., 1988) was supported by an easy standard format of a spreadsheet, together with an automatic support of calculations of all needed derivatives by an automatic differentiation program. However, IAC-DIDAS-N was a closed system, not easy to modify for specific classes of models being solved. This motivated the development of a 
modular system called IAC-DIDASN ++ , composed of modular tools written in the $\mathrm{C}++$ language.

A model in IAC-DIDASN++ is prepared as an ASCII text in a Model Description File format. The model can be purely nonlinear or consist of a linear and nonlinear parts. At present, the linear part can be expressed in the form of MPS file. Equations appearing in the nonlinear part must be explicitly resolvable. In this system there is no spreadsheet. A separate module called Model Compiler reads the file with model description, verifies its correctness and completeness, and translates it into forms suitable for other modules. This includes a representation of model variables and texts of procedures for efficient calculation of model outputs and their Jacobians. The Model Compiler produces a binary file with the definitions of all model variables and parameters and a $\mathrm{C}++$ code with procedures for the calculation of nonlinear functions and their Jacobians. This code can be compiled and linked with other modules. The binary file can be edited by Model Editor. The user can view inputs, outputs, parameters, lower and upper bounds, initial values as well as edit some of them.

The second set of tools provided in IAC-DIDASN ++ and adaptable for other similar systems concerns various modelling tasks such as direct simulation, inverse simulation, analysis of ranges on input and output variables, parametric and sensitivity analysis, single criteria optimization and multiobjective analysis; such tools include also specialized optimization solvers adaptable for these modelling tasks.

Various tools are provided for data analysis in graphic form as well as for graphic interaction between an user and the model analysis system. Other application specific tools can be added due to open architecture of the system. Moreover, dynamic interaction between user and the system gives additional possibilities to the user. For example, when the aspiration led methodology is applied to solving multiobjective optimization problems, the specification of reference levels is usually imprecise. The imprecision of reference levels specification can be included the in mathematical formulation of the decision problem by using fuzzy sets. The membership functions might be defined and modified graphically in such a case.

Together, all these tools constitute a modular environment for model analysis, optimization and decision support. Such environment is represented by two more specific cases: the IAC-DIDASN + + system for nonlinear models (described in this paper) and a similar system developed in cooperation with IIASA and other group of Polish researches, based on MOMIP solver for mixed integer programming models (Makowski, 1994). However, other systems for analyzing various classes of models can be also developed when using such an environment.

\section{STRUCTURE OF THE IAC-DIDASN++}

The IAC-DIDASN + + system is an open one - it consists of a set of independent modules with well defined interfaces, see Figure 1. Each module can potentially be exchanged and replaced by a another one. The system consist of the following modules:

- the system management module;

- model compiler; 


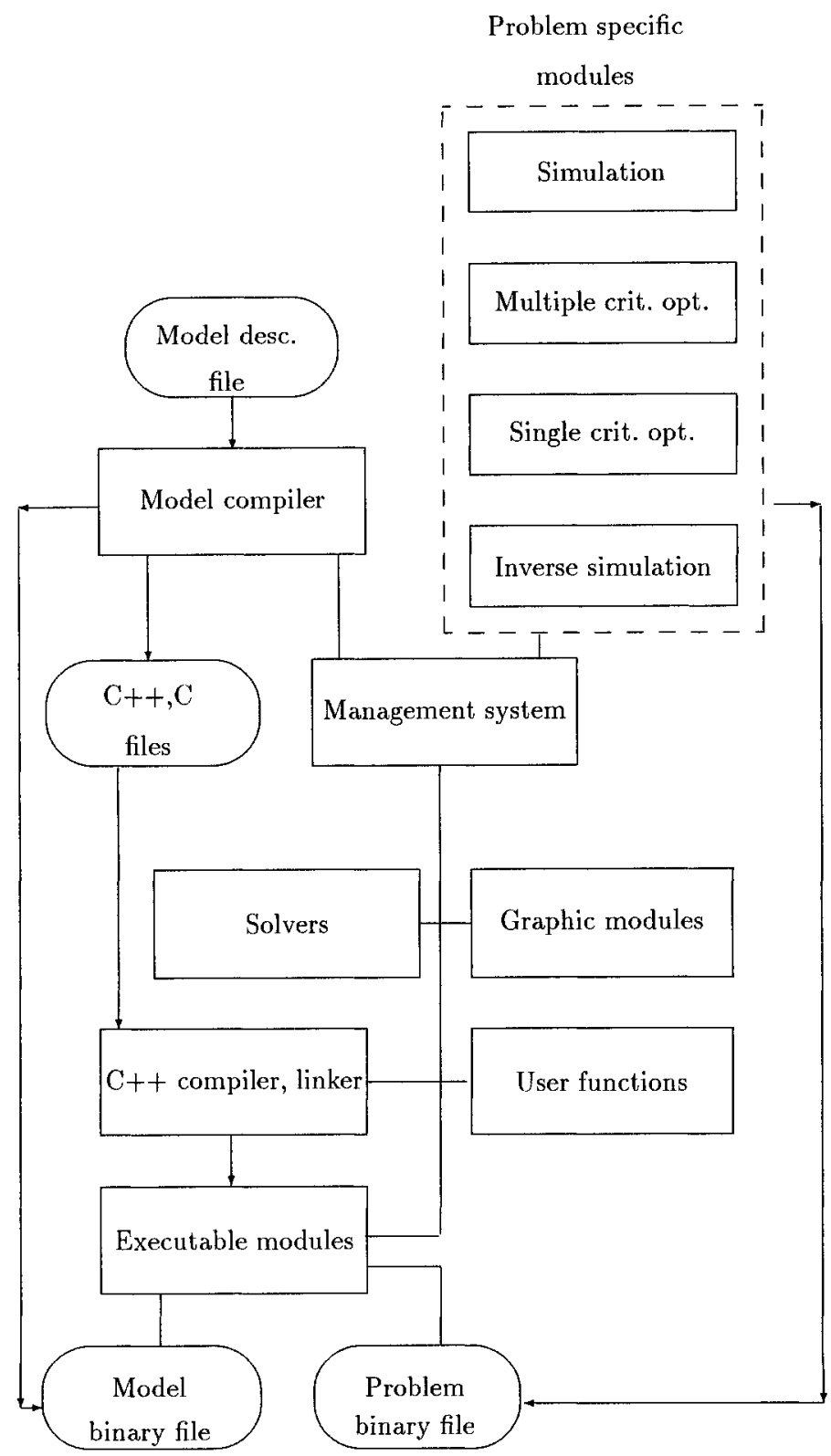

Figure 1 The structure of the IAC-DIDASN++ 
- model editor;

- problem editor;

- model debugger;

- modules to control the various optimization tasks;

- differentiable solver;

- nondifferentiable solver;

- graphical modules.

These modules can be divided into three groups:

- Executable modules: the system management, model compiler, model editor, problem editor.

- Modules which have to be linked with the models: model debugger, modules to control various optimization task.

- Modules, which are provided in the form of the libraries and could be used in the presented system as well as a tools in different systems: solvers, graphical modules.

The IAC-DIDASN ++ system was written in $\mathrm{C}++$ and developed on the Sun workstation under the operating system Solaris. The modules have been compiled with the gnu $\mathrm{C}++$ compiler. The OpenLook widget set was used to develop graphical user interface . It was verified that the whole system can be used on IBM-PC computers working under Solaris x 86 operating system. Some of the modules can also be run on an IBM-PC under MS-DOS. These modules were compiled with Borland $\mathrm{C}++$. The $\mathrm{C}++$ compiler must be installed on the user's computer since the $\mathrm{C}++$ code of the model file must be compiled and linked with other modules.

\section{THE MODEL FORMULATION AND INTRODUCTORY MODEL ANALYSIS}

The models considered in IAC-DIDASN ++ are nonlinear and can be expressed by the following format:

$$
\begin{aligned}
& \mathrm{y}_{1}=\mathbf{N}\left(\mathrm{x}_{1}, \mathbf{z}, \mathrm{y}_{1}\right)+\mathbf{A}_{11} \mathrm{x}_{1}+\mathbf{A}_{12} \mathrm{x}_{2}+\mathbf{B}_{1} \mathbf{z} \\
& \mathrm{y}_{2}=\mathbf{A}_{21} \mathrm{x}_{1}+\mathbf{A}_{22} \mathrm{x}_{2}+\mathbf{B}_{2} \mathbf{z} \\
& \mathbf{x}_{\mathbf{l}} \leq \mathbf{x} \leq \mathbf{x}_{\mathbf{u}} \\
& \mathbf{y}_{\mathbf{1}} \leq \mathbf{y} \leq \mathbf{y}_{\mathbf{u}} \\
& \mathrm{z}_{\mathbf{l}} \leq \mathrm{z} \leq \mathrm{z}_{\mathrm{u}}
\end{aligned}
$$

where

$$
\begin{array}{ll}
\mathbf{x} \in R^{n} & \text { - are decision variables } \mathbf{x}=\left(\mathbf{x}_{\mathbf{1}}, \mathbf{x}_{\mathbf{2}}\right) \\
\mathbf{z} \in R^{p} & \text { - are parameters } \\
\mathbf{y} \in R^{m} & \text { - are outcomes, } \mathbf{y}=\left(\mathbf{y}_{1}, \mathbf{y}_{\mathbf{2}}\right) \\
\mathbf{x}_{\mathbf{1}}, \mathbf{y}_{\mathbf{1}}, \mathbf{z}_{\mathbf{1}} & \text { - are lower bounds } \\
\mathbf{x}_{\mathbf{u}}, \mathbf{y}_{\mathbf{u}}, \mathbf{z}_{\mathbf{u}} & \text { - are upper bounds }
\end{array}
$$


A model is prepared in a Model Description File (MDF). The model can be purely nonlinear or consist of a linear and of a nonlinear parts. At present the linear part can be expressed in the form of a MPS file, which consist a fragment of the MDF or is included from it. Equations appearing in the nonlinear part must be explicitly resolvable. However, they can be introduced in any order as they are automatically sorted.

The Model Compiler is a tool for convenient formulation of mathematical models, their verification and automatic transformation to the form accepted by the IAC-DIDASN++ solver. The Model Compiler verifies the correctness of both the linear and nonlinear parts and produces:

- a binary file with the definitions of all model variables and parameters;

- a text file with procedures for the calculation of nonlinear functions and their gradients (i.e. outcomes of a nonlinear part of the model and their Jacobians);

- an (auxiliary) text file with the summary of contents of the binary file;

- a $\log$ file containing documentation of the compilation process, plus additional information, warnings and error messages.

The first two files are used by other modules.

Only original Model Description File is platform-independent, i.e. it can be transferred both between different operating systems (MS-DOS and UNIX) and different architectures under UNIX (Intel 386 and SUN Sparc).

A model description is contained in a text file called the Model Description File (MDF). Its general layout resembles slightly a classic MPS file format. Generally, a model can consist of a 'linear' and a 'nonlinear' part. The 'nonlinear part' means here - expressed in the form suitable for nonlinear models. In fact it can be linear, but the model compiler and the solver will treat it as a nonlinear one.

The syntax used presents a compromise between the orthodox MPS format and a 'free style' used in modern programming languages. The first parts of MDF, which are pertinent to the linear part inherit the MPS "by rows" structure. Its syntax is however relaxed slightly. There are no restrictions as to starting position of fields and their length. As a consequence of this the names used must be 'proper' in the typical programming sense, e.g. Vit.A is incorrect since it contains the dot.

The idea of defining nonlinear problems by extension of the MPS file was applied in the LANCELOT (Conn at al., 1992) system. Their SIF (Standard Input Format) adheres more closely to the MPS file including some of its rather archaic features.

The typical contents of a MPS file can be explicitly written into the MDF file - hence the restrictions forced by compatibility reasons. However there is another, perhaps more convenient form. An external MPS file can be simply included from the MDF file.

The nonlinear sections (if present) appear after linear section (if present). The "by rows" principle is abandoned and its contents can be edited in any reasonable fashion - subject to aesthetical limitations only.

Nearly all MDF key words have synonyms. They are introduced in order to allow a user to choose a form he/she is accustomed to. Key words in the linear and nonlinear parts are (with the common sense limitations) distinct, e.g. the identifier Rows has no predefined meaning in the nonlinear part. Maximal freedom is assured as to the usage or omission of 
terminators or/and separators and the order of appearance of many language elements. However the use of a consistent style is encouraged.

A model file consists of the following sections:

NAME, ROWS, COLUMNS, RHS, RANGES, BOUNDS, PARAMETERS , VARIABLES, EQUATIONS, ENDATA

The simple model:

$\min y=a *\left(x_{2}-x_{1}^{2}\right)^{2}+\left(x_{1}-1\right)^{2}$

with bounds:

$-10 \leq x_{1} \leq 10, \quad-10 \leq x_{2} \leq 10, \quad-100 \leq y \leq 100$

can be expressed by the following MDF:

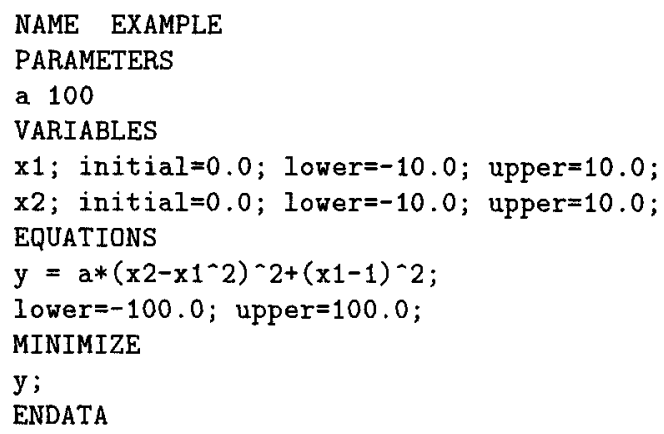

The Model Debugger can be used for investigation of the numerical properties of nonlinear models. The Problem Editor is a tool for the creation or modification of optimization problems. The user can view, define and modify optimization status of chosen outcomes.

\section{THE VARIOUS MODELLING TASK}

The following experiments are useful during the model analysis:

- direct simulation, including automatic calculation of the derivatives, step by step calculation of the model;

- analysis of ranges on the input and output variables as well as derivatives;

- single objective optimization and multiobjective analysis;

- parametric and sensitivity analysis;

- model inversion i.e.:

$\min _{\mathbf{x} \in X}\left\|\tilde{\mathbf{y}}-\tilde{\mathbf{y}}_{\text {ref }}\right\|+\rho\left\|\tilde{\mathbf{x}}-\tilde{\mathbf{x}}_{\text {ref }}\right\|$

subject to

$\mathbf{y}=\mathbf{F}(\mathbf{x}, \mathbf{z}, \mathbf{y}), \quad \mathbf{F}: R^{n} \times R^{p} \times R^{m} \mapsto R^{m}$

$\mathbf{x}_{\mathbf{l}} \leq \mathbf{x} \leq \mathbf{x}_{\mathbf{u}}, \quad \mathbf{y}_{\mathbf{l}} \leq \mathbf{y} \leq \mathbf{y}_{\mathbf{u}}, \quad \mathbf{z}_{\mathbf{l}} \leq \mathbf{z} \leq \mathbf{z}_{\mathbf{u}}$

$x_{i, r e f}: i \in I=\{1, \ldots, n\}, \quad y_{i, r e f}: i \in J=\{1, \ldots, m\}$ - the reference values for the selected outcomes and the decision variables. 
The optimization in IAC-DIDASN ++ is performed by a specially developed nonlinear optimization algorithm called solver. Since this maximization is performed repetitively, at least once for each interaction with the user that changes the parameters, there are special requirements for the solver that distinguish this algorithm from typical nonlinear optimization algorithms. It should be robust, adaptable and efficient, that is, it should compute reasonably fast an optimal solution for optimization problems of a broad class without requiring of the user to adjust special parameters of the algorithm in order to obtain a solution. The experience in applying nonlinear optimization algorithms in decision support systems (see Kreglewski at al., 1988) has led to the choice of an algorithm based on penalty shifting technique and projected conjugate gradient method. The algorithm have been used in IAC-DIDAS-N system. In the present system there is a modified version implemented in $\mathrm{C}$ language. The experimental nondifferentiable optimization solver is also provided (Granat at al., 1994).

\section{GRAPHICAL MODULES}

There are three different aspects of graphical information presentation which are present in IAC-DIDASN++:

- Presentation of results in graphic form to improve understanding of these results as well as of relationships between components of the decision problem being solved (objectives, decision variables etc.).

- Providing support for dynamic interaction between user and Decision Support Systems during specification of reference levels. This function contributes to the learning process by allowing an analysis of the history of interaction which has resulted in achieving the current state of the decision making process.

- A possible use of graphic interaction during the specification of reference levels, together with an interpretation of multiple reference levels (aspiration and reservation, possibly other levels) in terms of fuzzy set theory.

The IAC-DIDASN++ provides two graphical modules in the form of libraries. These modules supports the phase of interactive analysis and comparison of results phase in the case of multiobjective optimization.

The first module is used for specifying preferences of the user in terms of fuzzy sets. For each of the objective functions the linear membership function is presented. The user can interactively change membership functions by moving characteristic points. The thick lines show the sensitivity of the solution to the changes of the membership function for the objective. The detailed description can be found in (Granat and Wierzbicki, 1994).

The second one is used for specifying aspiration and reservation levels by using bar charts. The interactive screen consists of two window. The upper one presents the history of the process of interaction. The second one presents the current state of the objectives. For each of the objectives the following information is displayed: the solution (in the form of bar, and the numerical value), the aspiration level (the green triangle and the numerical value), the reservation level (the red triangle and the numerical value), the 
upper and the lower bounds (the numerical values). The user can change aspiration and reservation levels by moving the red and green triangles or by editing the numerical field.

\section{CONCLUSIONS}

The IAC-DIDASN ++ system have been applied for solving engineering problems in mechanics, automatic control and ship navigation (Wierzbicki at al., 1995) as well as for modelling environmental problems. The modular structure is especially useful for solving problems with real data. Usually in such cases the software should be customized for the specific problem being solved. Under development there is a new version of algebraic processing software and model compiler taking into account dynamic nonlinear models.

The development of the system was partly supported by the grant No. 883039102 and No. 0958/P4/94/06 of the Committee for Scientific Research of Poland.

\section{REFERENCES}

Brooke, A., Kendrick, D. and Meeraus, A. (1988) GAMS: a Users's Guide. The Scientific Press. Redwood City. USA.

Conn, A.R., Gould, N. and Toint, Ph. (1992) LANCELOT. A Fortran Package for LargeScale Nonlinear Optimization. Springer-Verlag. Berlin Heidelberg.

Granat, J. and Wierzbicki, A.P. (1994) Interactive Specification of DSS User Preferences in Terms of Fuzzy Sets. Working Paper of the International Institute for Applied Systems Analysis, WP-94-29, Laxenburg, Austria.

Granat, J., Kreglewski, T., Paczyński, J. and Stachurski, A. (1994) IAC-DIDAS-N++ Modular Modeling and Optimization System. Part I: Theoretical Foundations, Part II: Users Guide. Reports of the Institute of Automatic Control, Warsaw University of Technology, March 1994, Warsaw, Poland.

Kręglewski, T., Paczyński, J., Granat, J. and Wierzbicki, A.P. (1988) IAC-DIDAS-N: A Dynamic Interactive Decision Support System for Multicriteria Analysis of Nonlinear Models with a Nonlinear Model Generator Supporting Model Analysis. Working Paper of the International Institute for Applied Systems Analysis, WP-88-112, Laxenburg, Austria.

Lewandowski, A., Grauer, M., Wierzbicki, A.P. (1983). DIDAS: theory, implementation, in Interactive Decision Analysis (eds. M. Grauer, A.P. Wierzbicki), Proceedings Laxenburg 1983. Springer Verlag, Berlin Heidelberg (Lecture Notes in Economic and Mathematical Systems 229).

Makowski, M. (1994) Methodology and a Modular Tool for Multiple Criteria Analysis of LP Models. IIASA WP-94-102. Laxenburg. Austria.

Wierzbicki, A.P. and Granat, J. (1995) Multi-Objective Modeling for Engineering Applications in Decision Support. Paper presented at the Twelfth International Conference on Multiple Criteria Decision Making. Hagen. Germany. 